\title{
Use of an abdominal reapproximation anchor system in the closure of large, open myelomeningoceles
}

\author{
Patrick K. Jowdy, MD, ${ }^{1}$ Robert J. Perry, MD, ${ }^{2}$ and Renee M. Reynolds, MD ${ }^{1}$ \\ 'Department of Neurosurgery, Jacobs School of Medicine and Biomedical Sciences, University at Buffalo; and 'Division of Plastic \\ Surgery, University at Buffalo, New York
}

\begin{abstract}
Open neural tube defects are complex congenital abnormalities of the nervous system in which nervous tissue is exposed on the patient's back at the time of delivery. These malformations require surgical correction, and although replacement of the neural placode internally is fairly standard, providing skin closure can be very challenging especially in large defects. An abdominal reapproximation anchor (ABRA) device may be of value in attaining skin closure in these large, open myelomeningocele defects in which primary closure cannot be accomplished surgically. In a study period during which 65 patients underwent surgical closure of open myelomeningocele defects, 5 of them underwent ABRAassisted closure.

The average surgical intervention for myelomeningocele repair occurred on day 2.2 of life (range 2-3 days). The average defect size was $37 \mathrm{~cm}^{2}$ (range 16-56 cm $\mathrm{cm}^{2}$ ), and the average time to formal closure was 30.8 days (range 8-63 days). One of the patients had natural closure with just ABRA approximation. The remaining 4 patients underwent formal delayed primary closure when the skin edges became approximated. Use of the ABRA system in the closure of a large, open myelomeningocele may be valuable in select patients but requires further follow-up and comparison to identify truly significant differences with traditional techniques.
\end{abstract}

https://thejns.org/doi/abs/10.3171/2019.11.PEDS19225

KEYWORDS myelomeningocele; skin closure; ABRA device; congenital; surgical technique

$\mathrm{T}$ HREE of 10,000 live births in the United States are affected by spina bifida each year., ${ }^{1,7}$ Myelomeningocele, the most severe presentation of spina bifi$\mathrm{da},{ }^{4}$ is a complex congenital malformation of the nervous system that occurs early in gestation. This malformation results in nervous tissue being exposed on the patient's back at the time of delivery. Myelomeningocele defects can occur anywhere along the neural axis. They are most commonly seen in the lumbosacral region, and although these malformations vary in size and severity, all affect the integrity of the skin and nervous system with associated limitations in nervous system function.

Although replacement of the neural placode internally is fairly standard, providing skin closure can be very challenging especially in large defects. Given the spectrum of severity in terms of defect size and the influencing factors of location, shape, and surrounding soft tissue, several techniques have been developed between neurosurgeons and plastic surgeons to provide adequate skin defect closure. These include primary closure, skin advancement following extensive undermining of the subcutaneous plane, and a variety of skin flap and grafting techniques, which are usually reserved for larger defects over $5 \mathrm{~cm}$. In cases with larger defects necessitating more advanced closure techniques, the patient is at risk for postoperative complications with wound healing, with rates of skin flap necrosis, wound infection, and dehiscence between $18 \%{ }^{6}$ and $27.7 \%{ }^{10}$ reported in the literature.

In this article, we describe an innovative approach to the closure of extensive myelomeningocele defects utilizing a continuous dynamic tension device called the "abdominal reapproximation anchor" (ABRA) system (Canica Design). This system has been successfully employed in the plastic surgery community in the treatment of other large skin defects including abdominal wall defects, diabetic ulcers, and other soft-tissue defects secondary to trauma. ${ }^{3,8,9,11-13}$ To our knowledge, this report describes the first use of the ABRA system in myelomeningocele closure, an alternative technique to the current closure options available. 


\section{Methods}

This study is an institutional review board-approved (University at Buffalo Institutional Review Board), singleinstitution retrospective analysis of patients born with large, open myelomeningocele defects managed with the ABRA system in the period between January 1, 2000, and March 30, 2019. Patients were identified from the institutional Management Efficiency Doctor Efficiency Networked Together (MEDENT) electronic medical system using the ICD-9 and ICD-10 codes for myelomeningocele.

The practice at our institution for managing patients with open myelomeningocele includes delivery by Cesarean section and admission to the neonatal intensive care unit, after which neurosurgical consultation occurs. All patients are kept prone, with a moist semi-sterile dressing applied to the defect at all times. Prophylactic antibiotics including ampicillin and gentamicin are administered in all patients. Initial evaluation includes assessment for associated hydrocephalus, evaluation of neurological and urological function, and assessment of the defect size and configuration. All patients are scheduled within 72 hours for operative closure of the defect, and plastic surgery assistance is arranged for large defects, which can require more complex closure per the initial screening. Patients with diagnosed hydrocephalus undergo planned concurrent shunt placement in the same setting, which is done prior to defect closure while taking care to protect the defect during positioning and the surgical procedure. Following shunt placement or in those who do not require shunt placement, the patient is positioned prone with gel chest, hip, and head supports. The protective dressings are removed, and wide skin preparation occurs from the shoulders to the inferior thighs and laterally to the abdominal wall. The placode is released circumferentially and approximated into a tubular structure, followed by harvest of the laterally splayed dura and dural closure around the placode (Fig. 1).

During the study period, there was no specific published protocol for the management of myelomeningocele defect skin closure. Thus, at the time of surgery, skin closure techniques were based on the discretion of the treating pediatric neurosurgeon and any pediatric plastic surgical consultation. If primary skin closure was feasible, it was the preferred method. If it was not feasible, alternative techniques were implemented based on a combination of features including defect size, skin mobility, skin integrity, and defect location and in some patients included an ABRA-assisted closure. With this latter technique, the fascia was approximated where feasible, and extensive subcutaneous undermining in a deep subcutaneous plane over the lumbodorsal fascia to the posterior axillary lines was performed. Paired ABRA devices were then placed across the residual defect, with associated elastomers under tension. The defects were covered with IntraSite hydrogel (Smith \& Nephew) and Telfa (Medtronic; Fig. 2). Patients were kept prone postoperatively. Local wound care continued with twice daily IntraSite hydrogel replacement, and progressive elastomere tightening was performed based on the evaluation of the pediatric plastic surgeon. This continued until natural closure and healing were achieved or until the skin edges were in close enough approxima-

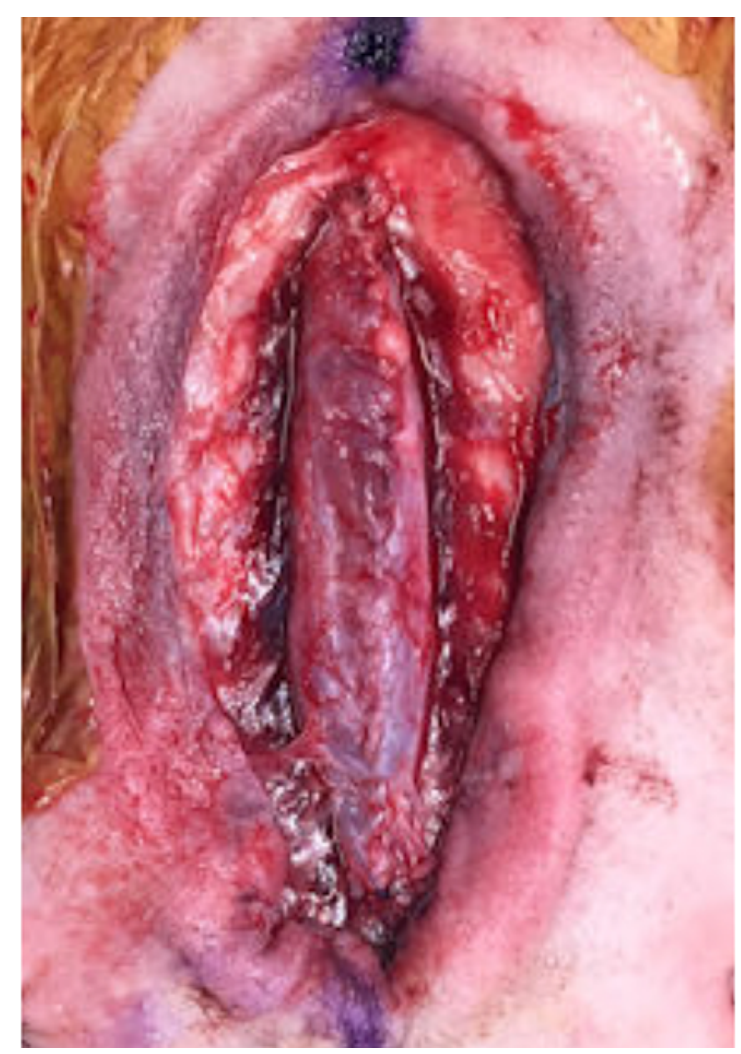

FIG. 1. Defect following placode reapproximation and dural closure. Figure is available in color online only.

tion that formal primary closure techniques could be implemented (Fig. 3).

\section{Results}

During the study period, 65 patients underwent surgical closure of open myelomeningocele defects. Of these patients, 10 required the assistance of a plastic surgeon for closure of the defect, and 5 underwent ABRA-assisted closure between April 2, 2014, and March 12, 2019. The average surgical intervention occurred on day 2.2 of life (range $2-3$ days). The average defect size was $37 \mathrm{~cm}^{2}$ (range 16$56 \mathrm{~cm}^{2}$ ), and the average time to formal closure was 30.8 days (range 8-63 days; Table 1). No patients were lost to follow-up, and the average follow-up was 2.71 years (range $0.44-5.08$ years). One of the patients had natural closure with just ABRA approximation. The remaining 4 patients underwent formal delayed primary closure when the skin edges became approximated. Four of the 5 patients underwent placement of a ventriculoperitoneal shunt, 2 at the time of initial closure and 2 in a delayed fashion. The decision for shunt placement was made secondary to a combination of features including head circumference, sutural splaying, and imaging features of ventricular enlargement. Neither of the delayed shunt placements was associated with cerebrospinal fluid leakage at the primary closure site. The average time to discharge was 40.6 days (range 10-76 days). The delay between the time of wound closure and discharge was secondary to parental teaching 


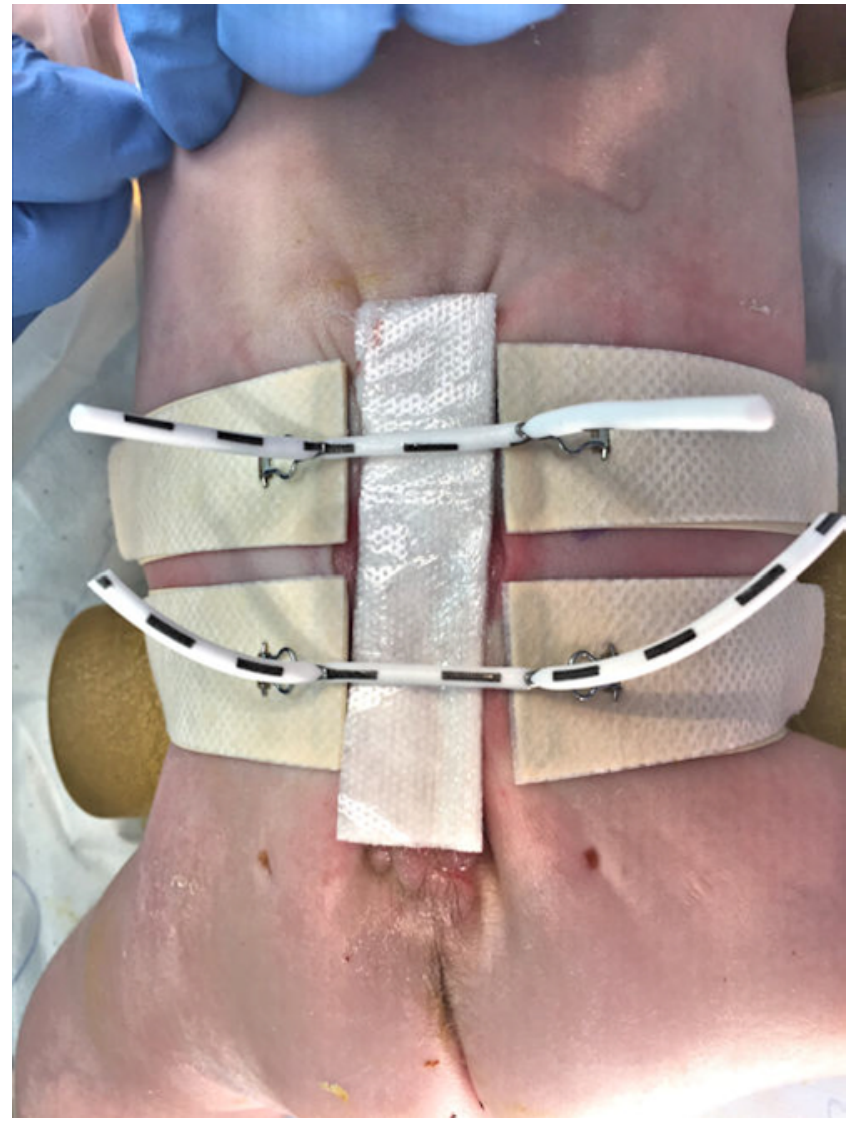

FIG. 2. After placement of IntraSite gel (Smith \& Nephew), Telfa (Medtronic), and the ABRA device (Canica Design). Figure is available in color online only.

in 2 patients and medical comorbidities that required additional treatment in the remaining 2 patients, including rectal prolapse necessitating ostomy in 1 patient.

There were no direct complications secondary to the ABRA-assisted closure. All patients had defect closure with the technique. There were no instances of skin necrosis, wound infection, wound breakdown, or cerebro- spinal fluid leakage. Two patients experienced unrelated complications including a shunt infection following delayed shunt placement at 6 weeks of age, at which time the lumbar closure had been completed. This necessitated externalization, antibiotics, and replacement of the shunt at a later date. One patient also developed suspected bacteremia based on sepsis criteria and was treated with antibiotics, although no culture was positive. Although it is possible that the ABRA device closure contributed to the above complications through the temporary skin defect given the time course of the shunt placement and infection and the lack of positive cultures for bacteremia, the likelihood is low.

\section{Discussion}

The goal of surgical intervention is to replace the neural elements into a more native position within a dural sac as well as to provide skin-covered closure to the defect. Release of the nervous tissue and mobilization of a dural closure are relatively standard in terms of surgical technique. Closure of the cutaneous elements, however, can be much more challenging and problematic, particularly in defects that are large as well as those that are close to the lumbosacral region where skin for mobilization is less available. Plastic surgeons are often involved in these complex closures and have developed a variety of techniques to provide cutaneous coverage. These include epidermal undermining, skin grafting, skin flap creation with rotation, transpositional or bipedicle techniques, and musculocutaneous flaps. ${ }^{6}$ Selection of any one technique is based on the features of the defect and which technique will best provide a tension-free and well-perfused covering. These techniques often require additional extensive incisions, which create additional scarring, cosmetic challenges, pain, and recovery. Despite meticulous techniques, there is a high rate of skin necrosis, with a failure rate up to $16 \%$ in cases with skin grafting, infection, and other wound healing challenges including granuloma formation. ${ }^{5}$ Moreover, these techniques often leave a cosmetically unappealing and debilitating abnormality at the closure site as well as the site of additional skin recruitment.

The ABRA is a dynamic closure system developed to
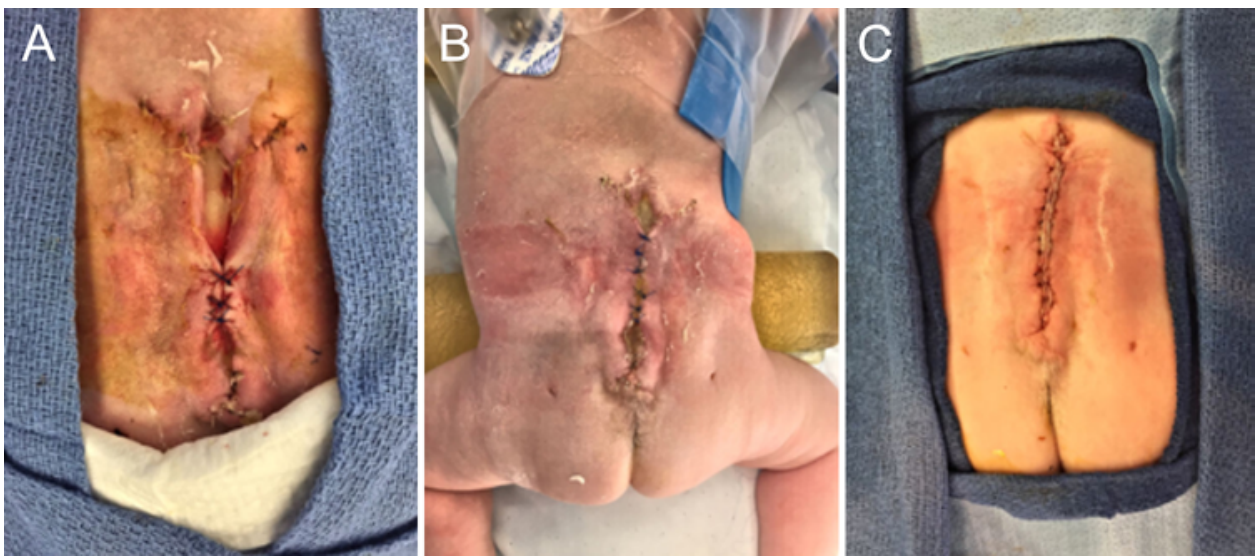

FIG. 3. Progressive traction (A and B) and subsequent delayed primary closure (C). Figure is available in color online only. 
TABLE 1. Summary of data in 5 patients who underwent ABRA-assisted closure of large, open myelomeningoceles

\begin{tabular}{|c|c|c|c|c|c|c|c|c|}
\hline $\begin{array}{l}\text { Case } \\
\text { No. }\end{array}$ & $\begin{array}{l}\text { Defect } \\
\text { Location }\end{array}$ & $\begin{array}{l}\text { Defect Size } \\
\quad(\mathrm{cm})\end{array}$ & $\begin{array}{l}\text { Days Until } \\
\text { Final Closure }\end{array}$ & $\begin{array}{l}\text { Days Until } \\
\text { Discharge }\end{array}$ & $\begin{array}{c}\text { Shunt } \\
\text { Placement }\end{array}$ & $\begin{array}{l}\text { Day of Life } \\
\text { Shunt Placed }\end{array}$ & Complications & $\begin{array}{l}\text { FU } \\
\text { (yrs) }\end{array}$ \\
\hline 1 & Lumbosacral & $4 \times 4$ & 13 & 28 & No & NA & $\begin{array}{l}\text { Suspected bacteremia } 14 \text { days } \\
\text { postop, culture negative }\end{array}$ & 5.08 \\
\hline 2 & Lumbar & $8 \times 6.5$ & 63 & 76 & Yes & 47 & Delayed shunt infection & 2.73 \\
\hline 3 & Lumbar & $6 \times 6$ & 52 & 63 & Yes & 249 & None & 3.73 \\
\hline 4 & Lumbosacral & $8 \times 7$ & 18 & 26 & Yes & 2 & None & 2.01 \\
\hline 5 & Lumbosacral & $5 \times 5$ & 8 & 10 & Yes & 2 & None & 0.44 \\
\hline
\end{tabular}

provide continuous tension on full-thickness, retracted skin defects, resulting in progressive wound closure and a sound delayed primary repair. The system comprises a variable number of anchoring devices depending on the size of the defect, which are adhered to the skin and subsequently placed under the tension of a pulley-elastomere unit that is designed to provide continuous but progressive tension on the wound edges, culminating in progressive skin edge approximation (Fig. 4). This device, originally developed to assist with the closure of large, high-tension abdominal defects, has been utilized in aiding closure in other large skin defects including diabetic ulcers and large traumatic defects and fasciotomy sites. ${ }^{3,8,9,11-13}$ Pressure sores related to the ABRA system have been reported as complications of the device by Verdam et al., but all healed without further complications. ${ }^{14}$ The benefits of this device over traditional techniques include the elastic nature of the device and the ability to avoid damage by evenly distributing force along the entire skin's edge, the eversion of the margins to avoid granulation tissue, the adjustable tension structure of the device to accommodate progressive tension and concerns with skin perfusion, and the documented ability to consistently achieve primary closure in

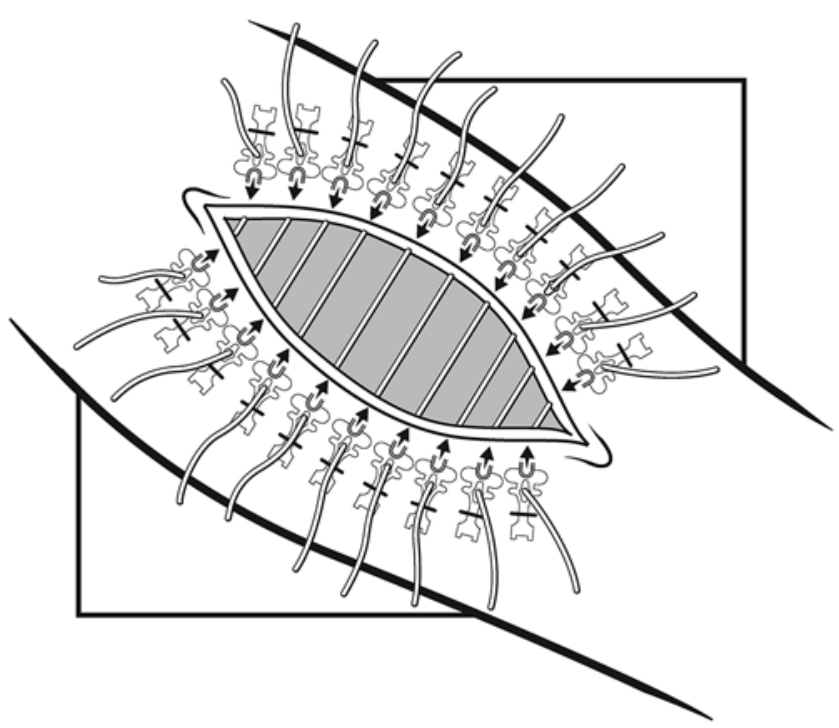

FIG. 4. Diagram of the ABRA surgical device with pulley-elastomere system. other sites. Secondary gains include the early promotion of patient mobility, a reduction in operating room visits, and a reduction in hospital-stay and home-care expenses.

To our knowledge, this is the first report on using the ABRA device in the closure of large, open myelomeningoceles, a unique population requiring complex wound closure techniques. In all 5 patients in whom the ABRA device was used, defect closure was attained. Although the number of patients was small, our success suggests that the device is at least as good as standard techniques. Additionally, as no directly associated complications were identified and the use of this device, as opposed to more complex flap techniques, limited patient exposure to additional extensive incisions, scarring, and pain, the ABRA system may be superior. "Additionally, although the financial implications in this patient population are not available, the utilization of the ABRA device for treatment of alternative conditions has demonstrated significant cost reduction including up to $\$ 28,000$ savings per patient when ABRA was utilized for fasciotomies.”2 By reducing rates of graft failure, wound healing challenges, and the other complications that accompany traditional closure techniques, the ABRA device may offer a financial benefit in the treatment of myelomeningocele. Long-term assessment with a larger patient cohort should be completed.

This study does have several limitations. It is a retrospective analysis of patients managed purely based on an individual surgeon's discretion of the defect and its optimal mode of closure. Additionally, without randomization, it is difficult to know whether classic techniques would have provided similar results. Lastly, a cost analysis is not presented, and use of the ABRA may in some cases extend hospitalization and overall costs. The purpose of this paper is only to make providers aware of an alternative technique that may be of value in select patients but requires further follow-up and comparison to identify truly significant differences with traditional techniques.

\section{Conclusions}

The ABRA device can be successfully employed in the closure of large, open myelomeningocele defects and can be considered in the armamentarium that plastic surgeons and neurosurgeons have to close these complex defects. Additional studies are necessary to further evaluate its effectiveness compared to current techniques as well as its cost-effectiveness. 


\section{Highlights}

- Providing skin closure for open myelomeningocele skin defects, especially large defects, can be very challenging.

- Many of the current techniques employed to provide skin closure can result in excessive tension on the skin, which affects local blood supply and leads to a high rate of wound healing challenges.

- The ABRA system is a traction device developed for open wounds with retracted skin defects, allowing progressive traction with chronic cyclic tension to provide sound delayed primary closure and showing in the plastic surgery literature its effectiveness in place of skin grafting.

- To our knowledge, this is the first documented use of the ABRA system in myelomeningocele closure, which can serve as an alternative to the current closure options available.

\section{Acknowledgments}

We thank W. Fawn Dorr, BA, for editorial assistance.

\section{References}

1. Boulet SL, Yang Q, Mai C, Kirby RS, Collins JS, Robbins $\mathrm{JM}$, et al: Trends in the postfortification prevalence of spina bifida and anencephaly in the United States. Birth Defects Res A Clin Mol Teratol 82:527-532, 2008

2. Clinician Corner: Dynamic Tissue Systems. ACell Inc. (https://acell.com/clinician-corner/) [Accessed November 25, 2019]

3. Heimes J, Carlton E, McDonnell J, Rogers T, Udobi K, Moncure M: Use of an abdominal reapproximation anchor (ABRA) system in a patient with abdominal compartment syndrome after severe burns: a case report. Burns 39:e29e33, 2013

4. Kellogg R, Lee P, Deibert CP, Tempel Z, Zwagerman NT, Bonfield CM, et al: Twenty years' experience with myelomeningocele management at a single institution: lessons learned. J Neurosurg Pediatr 22:439-443, 2018

5. Kemaloğlu CA, Özyazgan İ, Ünverdi OF: A decision-making guide for the closure of myelomeningocele skin defects with or without primary repair. J Neurosurg Pediatr 18:187-191, 2016

6. Kobraei EM, Ricci JA, Vasconez HC, Rinker BD: A comparison of techniques for myelomeningocele defect closure in the neonatal period. Childs Nerv Syst 30:1535-1541, 2014
7. Kshettry VR, Kelly ML, Rosenbaum BP, Seicean A, Hwang L, Weil RJ: Myelomeningocele: surgical trends and predictors of outcome in the United States, 1988-2010. J Neurosurg Pediatr 13:666-678, 2014

8. Mukhi AN, Minor S: Management of the open abdomen using combination therapy with ABRA and ABThera systems. Can J Surg 57:314-319, 2014

9. Price J, Rubens F, Bell M: Elastic device facilitating delayed primary closure of sternal wound infection. Ann Thorac Surg 83:1162-1165, 2007

10. Seidel SB, Gardner PM, Howard PS: Soft-tissue coverage of the neural elements after myelomeningocele repair. Ann Plast Surg 37:310-316, 1996

11. Singh N, Bluman E, Starnes B, Andersen C: Dynamic wound closure for decompressive leg fasciotomy wounds. Am Surg 74:217-220, 2008

12. Taylor RC, Reitsma BJ, Sarazin S, Bell MG: Early results using a dynamic method for delayed primary closure of fasciotomy wounds. J Am Coll Surg 197:872-878, 2003

13. Urbaniak RM, Khuthaila DK, Khalil AJ, Hammond DC: Closure of massive abdominal wall defects: a case report using the abdominal reapproximation anchor (ABRA) system. Ann Plast Surg 57:573-577, 2006

14. Verdam FJ, Dolmans DE, Loos MJ, Raber MH, de Wit RJ, Charbon JA, et al: Delayed primary closure of the septic open abdomen with a dynamic closure system. World J Surg 35:2348-2355, 2011

\section{Disclosures}

The authors report no conflict of interest concerning the materials or methods used in this study or the findings specified in this paper.

\section{Author Contributions}

Conception and design: all authors. Acquisition of data: all authors. Analysis and interpretation of data: all authors. Drafting the article: Reynolds, Jowdy. Critically revising the article: all authors. Reviewed submitted version of manuscript: all authors. Approved the final version of the manuscript on behalf of all authors: Reynolds.

\section{Correspondence}

Renee M. Reynolds: Jacobs School of Medicine and Biomedical Sciences, University at Buffalo, NY.rreynolds@ubns.com. 